\title{
Vascularization in skin wound healing: where do we stand and where do we go? Helena R Moreira ${ }^{1,2}$ and Alexandra P Marques ${ }^{1,2}$
}

Cutaneous healing is a highly complex process that, if altered due to, for example, impaired vascularization, results in chronic wounds or repaired neotissue of poor quality. Significant progress has been achieved in promoting neotissue vascularization during tissue repair/regeneration. In this review, we discuss the strategies that have been explored and how each one of them contributes to regulate vascularization in the context of cutaneous wound healing from two different perspectives - biomaterial-based and a cell-based approaches. Finally, we discuss the implications of these findings on the development of the 'next generation' approaches to target vascularization in wound healing highlighting the importance of going beyond its contribution to regulate vascularization and take into consideration the temporal features of the healing process and of different types of wounds.

\footnotetext{
Addresses

${ }^{1}$ 3B's Research Group, I3Bs - Research Institute on Biomaterials, Biodegradables and Biomimetics, University of Minho, Headquarters of the European Institute of Excellence on Tissue Engineering and Regenerative Medicine, Avepark - Zona Industrial da Gandra, Guimarães 4805017, Portugal

${ }^{2}$ ICVS/3B's - PT Government Associate Laboratory, Braga, Guimarães 4805-017, Portugal
}

Corresponding author:

Marques, Alexandra P. (apmarques@i3bs.uminho.pt)

Current Opinion in Biotechnology 2021, 75:253-262

This review comes from a themed issue on Tissue, cell and pathway engineering

Edited by Lorenzo Moroni and Martijn van Griensven

https://doi.org/10.1016/j.copbio.2021.08.019

0958-1669/๑ 2021 Elsevier Ltd. All rights reserved.

\section{Introduction}

Wound healing is a complex and highly regulated process, commonly organized in phases that include (i) hemostasis, (ii) inflammation, (iii) proliferation, and (iv) remodeling $\left[1^{\bullet \bullet}\right]$. Upon injury, intravascular platelets are activated and secrete a series of growth factors that act as chemoattractants to neutrophils and monocytes [2]. These differentiate into macrophages in the inflammatory phase [3] releasing cytokines (vascular endothelial growth factor (VEGF)-A, fibroblast growth factors (FGFs) and angiopoietins (Ang)) that stimulates fibroblasts migration and activates microvascular endothelial cells (ECs) [4]. Within a few days, angiogenic capillary sprouts invade the fibrin/fibronectin-rich wound clot and organize into a microvascular network due to the dynamic interaction that occurs among ECs, angiogenic cytokines and the extracellular matrix (ECM) environment produced by fibroblasts (Box 1, Figure 1) [5]. However, when the wound healing cascade is affected, as for example due to delayed vascularization, dysfunctional healing occurs impacting the quality of the neotissue formed. Moreover, in chronic wounds, the angiogenic process is impaired which, associated to a persistent inflammatory state, hinders healing progression. These wounds are associated to other co-morbidities such as diabetes and arterial and venous insufficiencies [6], being therefore the most challenging. For example, lower limb amputation due to diabetic foot ulcers occurs in $14 \%-24 \%$ of diabetes patients; while approximately $77 \%$ of those ulcers heal within 1 year, roughly $40 \%$ of patients have a recurrence within 1 year after healing, almost $60 \%$ within 3 years, and $65 \%$ within 5 years $[7,8]$. It is then clear that chronic wounds have a growing prevalence worldwide being a major health problem with important social and economic implications with an urge need for improved therapeutic approaches.

Significant progress has been achieved in promoting neotissue vascularization during tissue repair/regeneration [9]. Whether these have considered the needs of each cutaneous wound where a local and temporal effect must be attained to promote not only wound closure - open skin wounds might be life-threatening — but also the neotisue quality - directly associated to tissue functionality also critical for keeping body homeostasis - should be analyzed. In this review, the strategies that have been explored and how each one of them contributes to regulate vascularization in the context of cutaneous wound healing were analyzed from two different perspectives biomaterial-based and a cell-based approaches. We discuss the efforts that have been made to optimize biomaterials/scaffolds to have spatial, temporal or spatio-temporal control of angiogenesis/vascularization at wound site $[10,11]$ (Figure 2). Spatial regulation can be mostly associated to the target of local cells by presenting-specific peptides at the biomaterials surface that then drive integrin-mediated angiogenesis. On the other hand, regulation of angiogenesis along time is intrinsically linked to 


\section{Box 1 Vasculogenesis versus angiogenesis}

In adult skin, within the wound, neovascularization occurs through blood vessel formation via vasculogenesis or angiogenesis (Figure 1) [17]. Vasculogenesis - generation of new vascularization in the absence of preexisting blood vessels - takes place when EPCs home to the damaged site and differentiate into ECs to form primitive vascular networks [18]. However, in the context of wound healing, angiogenesis - formation of new blood vessels from pre-existing vasculature [19] - is the most common vascularization process. Sprouting angiogenesis, starts with an EC - tip cell - in an existing vessel that degrades the original basement membrane with a highly controlled proteolytic activity cleaving basement membrane and interstitial proteins. As a result, EC projects protrusions through the gap in the basement membrane into the interstitium leading a string of ECs that are able to proliferate and create a branch, increasing in complexity with the growth of the vascular network [18]. Although the angiogenesis process in adult cutaneous wound healing is wellestablished, the existence and the potential role of vasculogenesis process in this healing process is controversial and not yet clear [17]

angiogenic byproducts or via controlled release/delivery of angiogenic growth factors (GFs) or respective encoding genetic material. Regarding cell-based approaches, prevascularization of cellular tissue engineered constructs using both mature [12] and progenitor [13,14] ECs is one of the most successful approaches in fostering vascularization at wound site. However, the clinical use those cells is highly hampered by an insufficient number required for therapy due to senescence and impaired capacities for vascular repair associated to their expansion in vitro [15]. Knowing that ECs wound surroundings are key to the process of new vessels formation [16] we discuss how the angiogenic signals secreted by other skin cells, represent an opportunity to define new strategies capable of directing wound healing angiogenesis (Figure 3). Moreover, we discuss how adipose tissue has been contributing to promote the neovascularization of wounds focusing on the angiogenic secretome of ASCs and on the cellular complexity of adipose tissue stromal vascular fraction (SVF) highlighting its potential as an alternative to promote constructs pre-vascularization.

\section{Biomaterial-controlled vascularization in skin wound healing Spatial regulation}

Because of the recognition of biological cues by cell integrins, peptide-functionalized materials have been explored to promote adhesion of angiogenic cells, such as ECs and EPCs, fostering angiogenesis locally at the wound site. These cells recognize the underlying matrix through different sets of integrins, which are known to be involved in triggering signaling pathways related to migration, proliferation and organization in vascular networks [9,20]. Early studies showed that materials functionalized with the A13 and C16 sequence from laminin [21] and the RGD and the heparin-binding domain of fibrin $\left(\mathrm{Fg} \beta 15-66_{(2)}\right)$ [22] promoted higher EC migration and improved neovessel formation in full-thickness wounds. However, most of these peptides have no selectivity for angiogenic cells and therefore can mediate adhesion of other cells resulting in a competitive environment that can be detrimental for local angiogenesis. This was in fact demonstrated with alginate hydrogels

\section{Figure 1}

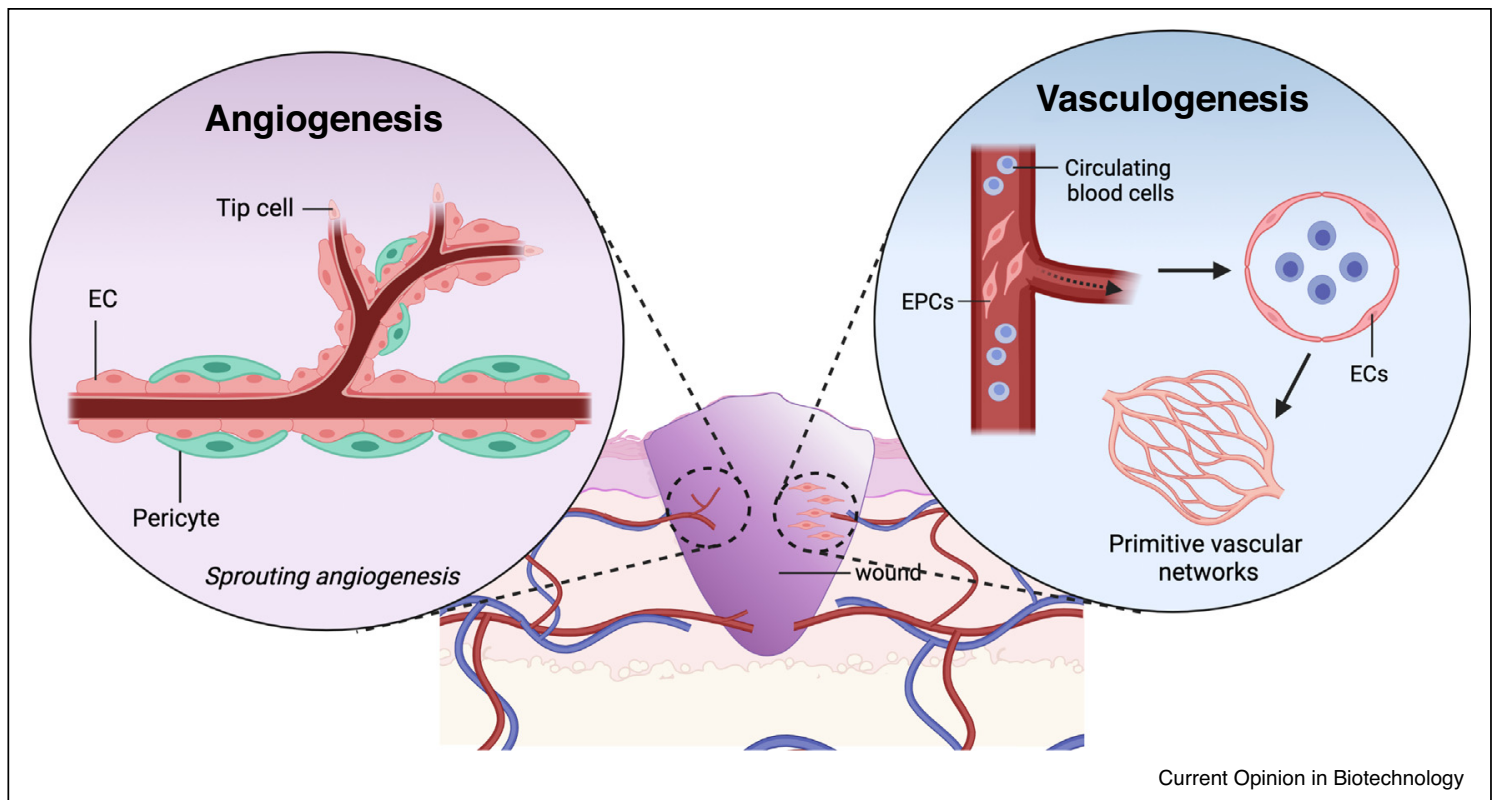

Vasculogenesis and angiogenesis in wound healing.

EC - endothelial cell; EPCs - endothelial progenitor cells. 


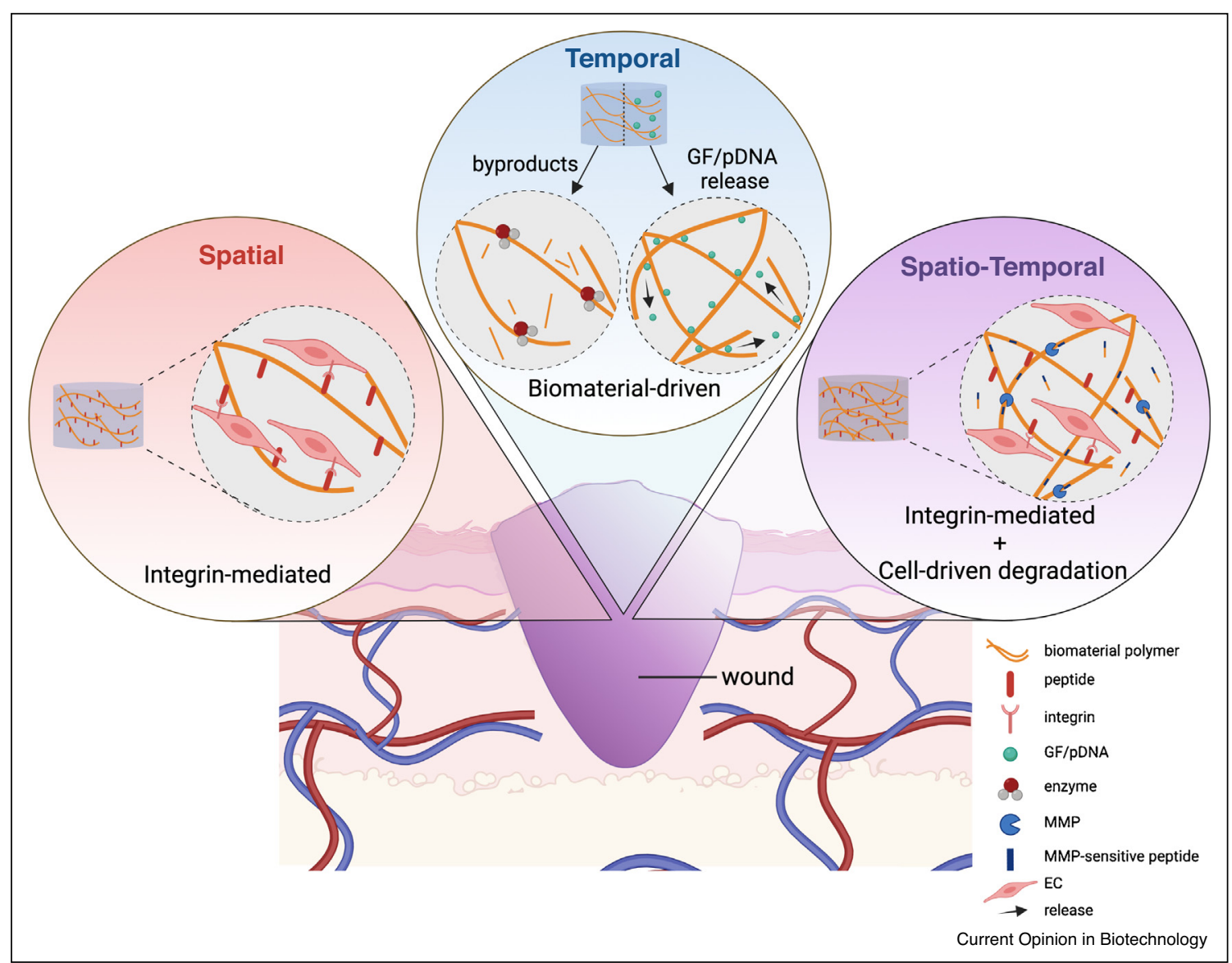

Biomaterial-based strategies to promote vascularization in wound healing.

EC - endothelial cell, GF - growth factor, MMP - metalloproteinase, pDNA - plasmid encoding DNA.

functionalized with peptides specific for $\alpha 4 \beta 1$ integrin. Materials with the REDV sequence showed superior capability to promote selective adhesion and proliferation of ECs in vitro and higher density of newly formed vessels after subcutaneous implantation in rats, than the ones with the RGD or the YIGSR peptides [23]. The importance of this specificity was recently reinforced with hyaluronic acid hydrogels functionalized with the fibronectin fragment (Fn9-10) that was designed to be specific for $\alpha 3 / \alpha 5 \beta 1$-integrin or $\alpha v \beta 3$-integrin [24 $4^{\circ}$. Matrices with the $\alpha 3 / \alpha 5 \beta 1$ led to the formation of non-leaky blood vessels in opposition those with specificity for $\alpha v \beta 3$ integrin, which was attributed to the higher expression of VE-cadherin at cell-cell junctions. Interestingly, RGD and $\alpha v \beta 3$-ligand functionalized matrices led to similar ECs sprouting and formation of disorganized structures however, this was observed only for a much higher concentration of RGD peptide $(>500 \mu \mathrm{M})$ than fibronectin fragments used $(2 \mu \mathrm{M})$.
While these approaches highlight the possibility of spatially control vascularization, whether they are effective in cutaneous wound healing is still to be demonstrated. Moreover, the fundamental understanding of integrinmediated responses to peptide-functionalized biomaterials, including concentration, and other neighboring ligands, and their engagement as angiogenic/vasculogenic initiators requires further investigation.

\section{Temporal regulation}

In processes like angiogenesis and vascularization, temporal ECM remodeling is crucial for vascular cell migration and cell-cell organization [9]. Therefore, biomaterials - ECM mimickers - that are susceptible to hydrolytic or enzymatic degradation are intrinsically potential regulators of temporal tissue response. It is long known that biomaterial degradation byproducts, such as those of fibrin [25,26] or hyaluronic acid [27 $]$, modulate the proliferation and migration of angiogenic cells. 


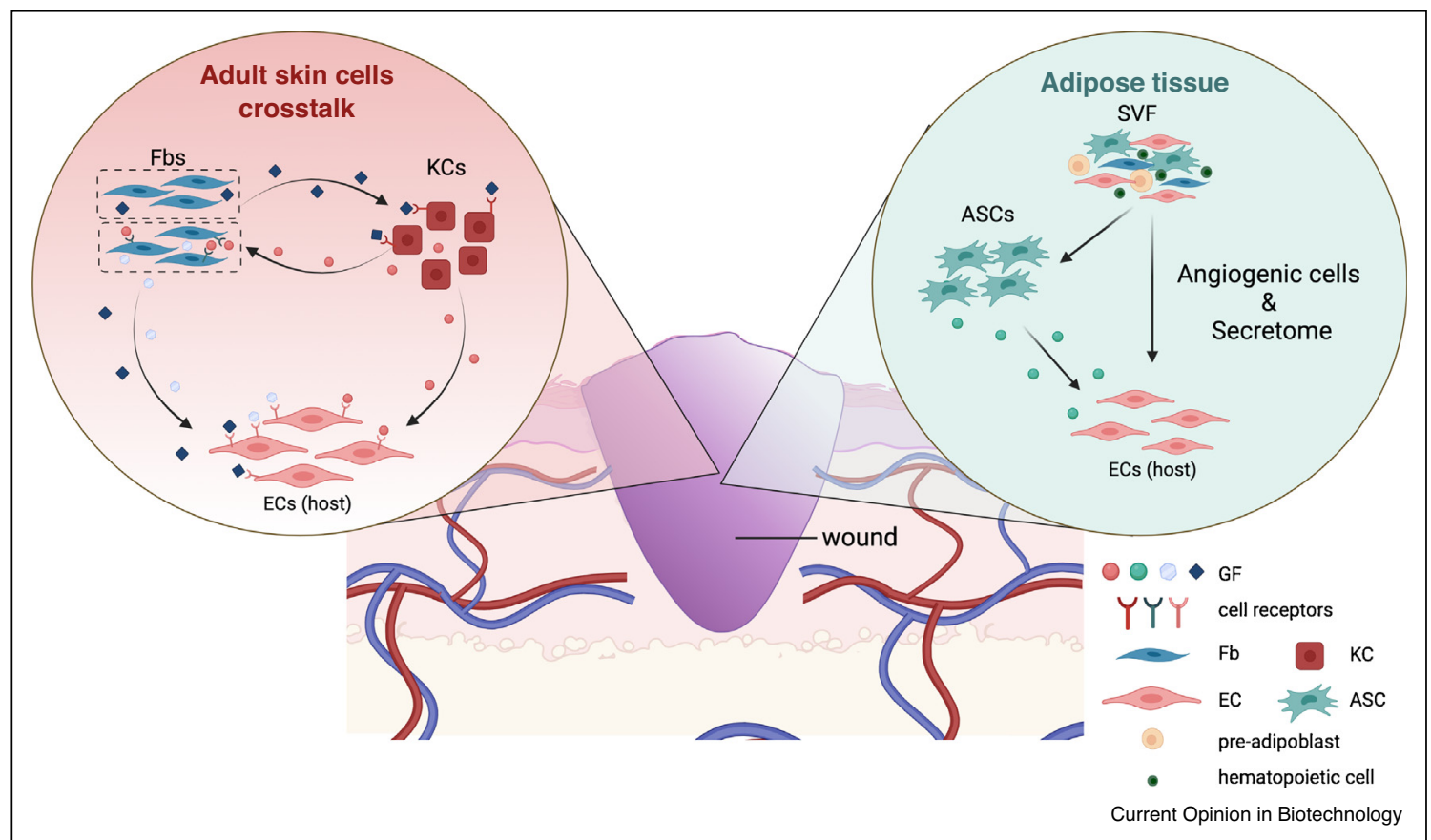

Cell-based strategies in skin wound healing vascularization.

ASC - adipose-derived stem cell, EC - endothelial cell, Fbs - fibroblasts, GF - growth factors, KCs - keratinocytes, SVF - stromal vascular fraction.

However, the temporal control of angiogenesis using these materials is limited by their degradation rates [28,29], being therefore mostly suited for addressing angiogenesis at early wound healing stages. The combination of these materials with others that are not degraded enzymatically has allowed to tackle this limitation. This is the case of hyaluronic acid and gellan gum structures that promoted the neovascularization of ischemic mice hind limb in a material-dependent manner, consistent with the in vitro degradation profile (up to 28 days) [27 $]$. These materials were also able to support significantly higher vessel density in diabetic mice full-thickness wounds along the whole healing timeframe [30].

While biomaterial degradation byproducts are a valid option, temporal regulation of angiogenesis during wound healing has been mostly associated to the biomaterialmediated delivery/release of biomolecules. These approaches take advantage of the kinetics of diffusion controlled through materials' bulk properties (polymer concentration, crosslinking degree, wettability), or the degradation rate of the matrices to which biomolecules are coupled to. Early studies showed that VEGF [31], basic FGF (bFGF) [32] and stromal-derived factor 1 (SDF-1) [33] delivered using fibrin matrices, gelatin sheets and decellularized dermis, respectively, enhanced the vascularization of full-thickness mice wounds.
Despite this, co-delivery of two GFs, being VEGF the prime angiogenic factor, seems to be more effective than their individual administration. Moreover, the use of angiogenic factors that are known to have specific roles at different times of the angiogenic process was considered. This was shown with a bioengineered mussel adhesive protein-based gel entrapping VEGF and platelet derived growth factor (PDGF)-containing microparticles in a full-thickness rat wound. While $40 \%$ of VEGF was release during one day, PDGF was release slowly over 4 days resulting in significantly higher capillary density and overall improved neotissue quality [34]. However, the link between the role of each one of those factors and the observed response was not made.

An extension of the delivery window was also considered via covalent immobilization, yet this has not been necessarily linked to a higher control of the delivery/release. Chitosan scaffolds functionalized with VEGF and bFGF via $\mathrm{PEG}$, shown to be sensitive to the action of lysozyme releasing in vitro up to $70 \%$ of the coupled GFs, led to enhanced vascularization of full-thickness rat wounds at early time-points but details on the temporal effect of the GFs were not provided [35]. Similarly, collagen functionalized with bFGF and keratinocyte growth factor (KGF) via EDC/NHS supported increased blood flow in a fullthickness rat wound between days 7 and 14 [36]. 
Interestingly, at day 14 this is significantly different from groups with single GFs which demonstrates an enhanced effect of the dual system. However, the blood flow profile along the time was similar to all the conditions which might correlate with the sustained release profile observed in vitro, similar for both GFs. In other approach, collagen scaffolds to which VEGF and SDF-1 $\alpha$ were coupled via a collagen binding domain, also led to the formation of a higher number of vessels in full-thickness wounds in diabetic rats. Interestingly, the mount of ECs recruited to the site of injury by scaffolds loaded with up to $20 \mu \mathrm{g}$ of VEGF was almost null while a dose-dependent response was observed for SDF- $1 \alpha$-containing scaffolds. Thus, this work seems to show that SDF-1 $\alpha$ is responsible for ECs recruitment that are then led by VEGF to organize and form the vessels [37]. Also, fibrin hydrogels containing VEGF-165 and PDGF-BB coupled via a high GFs affinity peptide (LAMA3 $3043-3067$ ), led to a significantly increased frequency of proliferative cells in full-thickness wounds in type-2 diabetic mice compared to other treatment groups at day five. This is consistent with an early effect of the co-delivery of those GFs that results in an accelerated healing [38 $8^{\circ}$. In this case, neovascularization might also have been potentiated by the synergistic effect of the fibrin byproducts although authors do not take this into consideration.

Despite what was achieved with the delivery of GFs, limitations that includes bolus release of proteins, short half-life due to lack of protection from proteolytic degradation, and the requirement of high doses to achieve a therapeutic effect [39], which in the case of angiogenic factors can be linked to tumor development [40] are still a reality. Biomaterials delivering genetic material encoding for specific angiogenic proteins have been showing to foster the sustained production of GFs by host cells. Gene-activated matrices (GAMs) or scaffolds rely on combining non-viral vector therapeutic genes within 3D structures that offer structural support and a matrix for new tissue deposition [41]. Up to now, collagen/chitosan scaffolds loaded with lipofectamine 2000/pVEGF transplanted into fullthickness wounds in rats lead to an upregulation of VEGF secretion that resulted in an increased number of newly formed vessels 9 days post-implantation than the structures with non-complexed pVEGF or with lipofectamine/pGFP [42]. Similarly, hyaluronic acid/ dextran hydrogels containing polyethyleneimine $(\mathrm{PEI}) / \mathrm{pVEGF}$ promoted microvessels formation in full-thickness rat burns, in a higher extent than the hydrogel alone due to the effective secretion of VEGF from the in situ transfected cells over 7 days [43].

Overall, independently of the strategy the challenge to control vascularization in cutaneous wound healing still relies on matching the exact temporal profile of delivery that responds to its evolution.

\section{Spatio-temporal regulation}

As discussed, biomaterial-based approaches are capable of individually impacting neovascularization both at spatial and temporal level. One might question if spatio-temporal strategies synergize towards this, being more successful in accelerating vascularization in wound healing. Biomaterials have been functionalized with peptides that are recognized by angiogenic cells promoting adhesion and act as sensitive biodegradation sites supporting, along time and depending on the degradative milieu, cell migration, proliferation and organization. In hyaluronic acid-modified hydrogels, EC migration throughout the matrices was enabled through the MMP-sensitive crosslinker (GCRDGPQG $\downarrow$ IWGQDRCG), and vacuole and lumen formation were RGD dose-dependent [44]. This organization was observed within 3-6 hours, while the cell migration was assumed to lead the creation of 'vascular guidance tunnels' that supported branching and sprouting after 2-3 days. In another approach, T1 (GQKCIVQTTSWSQCSKS) or C16 (CGGKAFDITYVRLKF) peptides linked to in gellan gum were shown to determine EC adhesion in a concentration-dependent manner, while the MMP-1 cleavable peptide (CRDGPQGIWGQDRC) allowed opening space in the polymeric network of the hydrogel for subsequent cell spreading $\left[45^{\circ}\right]$. Despite the modest results, it might be argued that the in vitro concentration of MMP-1 and the time of culture were not sufficient to further degrade the matrix degradation allowing ECs organization.

Overall, as precise spatial and temporal regulation of angiogenesis is necessary for enhanced wound healing and formation of neotissue with a quality closer to the native one, the combinatorial approach may be the best approach to mimic regulation of angiogenesis using biomaterials properties.

\section{Cell-controlled vascularization in skin wound healing}

\section{Dermal and epidermal cells signaling}

While poorly representing the complexity of native tissue, skin substitutes are in general effective in promoting wound healing. Dermal and dermal-epidermal products composed respectively by fibroblasts, and fibroblasts and keratinocytes, can however be associated to delayed engraftment mostly due to deficient neo-vascularization. It is also known that both keratinocytes and fibroblasts impact ECs, which seems to reveal that the potential of those cells as part of dermal-epidermal skin substitutes is not maximized. During the proliferation and remodeling phases of the wound healing, fibroblasts secretion of keratinocyte GF-1 (KGF-1), KGF-2 and interleukin-6 (IL-6) have a profound effect on migration, proliferation and differentiation of keratinocytes [46,47]. In response to these factors, keratinocytes are also prompt to secret VEGF, which guides angiogenesis in the wound tissue through the upregulation of nitric oxide (NO) in ECs [48]. 
This is in line with the enhanced wound vascularization observed after transplantation of skin substitutes containing keratinocytes genetically modified to overexpress VEGF [49,50]. Moreover, keratinocyte-to-EC crosstalk is known to be involved in wound angiogenesis. Impaired EC migration and angiogenesis was observed in epidermis-specific $\alpha 3$-integrin-knockout mice due to the reduced expression of the pro-angiogenic factor MRP3 by keratinocytes [51]. Therefore, novel signaling mechanisms may prospectively be targeted in keratinocytes to promote angiogenesis during wound-healing, particularly at the re-epithelialization stage.

The crosstalk between keratinocytes-fibroblasts-ECs is thought to be responsible for regulating microvascular density within the papillary and reticular dermis [52], being therefore also an axis of interest to target during wound healing. Keratinocytes secretome was shown to trigger the secretion of factors such as VEGF-A, FGFs, Ang-1 and hepatocyte growth factor/scatter factor (HGF/ $\mathrm{SF}$ ) by fibroblasts [53-55], known to induce EC migration, tubule formation and stabilization $\left[56,57^{\circ}\right]$. Additionally, a stiffer microenvironment formed by ECM proteins deposited by fibroblasts correlates with enhanced EG vessel sprouting with lumen formation [58]. It is also known that the capacity ECM has to retain specific molecules is also determinant for this fibroblastsECs crosstalk [59]. This is the case of the ECM produced by the papillary fibroblasts that supports the formation of a robust array of highly branched tube-like structures due to the capacity to link HGF/SF, known to be critical for EC differentiation [60]. While this differentiated capacity of fibroblasts to support ECs could be explored for cutaneous wound healing, the use of pure papillary and reticular fibroblasts is still dependent on the identification of specific surface for their isolation [61]. There are also other fibroblasts subpopulations that could be of interest to explore in this context [61], therefore, the subsequent elucidation of the role of each fibroblast subpopulation in angiogenesis is pivotal for their use as alternative to target vascularization.

\section{Adipose tissue as a cell source}

The adipose tissue constitutes a relevant source cells for tissue repair and regeneration due to a wide range of features [62]. The adipose-derived stem cell (ASCs), in particular, are known to secrete various growth factors, including VEGF-A, FGFs, transforming growth factor- $\beta$ (TGF- $\beta$ ), placental growth factor (PGF), and anti-apoptotic factors [63-65], which have lead their exploitation as key pro-angiogenic mediators. Increased neo-vascularization due to the paracrine interaction of the ASCs with host resident cells was in fact confirmed in full-thickness excisional mice wounds [66]. Increased blood flow in a third degree burn in sheep was also attributed to the VEGF secreted by the transplanted ASCs [67]. Similarly, the secretion of HSF by ASCs was also correlated with a significantly higher number of ECs in full-thickness mice ulcers [68]. This effect can be however dependent on the time of residence of the transplanted cells as shown by the secreted VEGF, Ang-1 and bFGF dose-dependent number of vessels formed at early time-points in a deep partial thickness burn in pigs [69]. This angiogenic effect of the ASCs was further shown to synergize with platelet-rich plasma (PRP) as revealed by the higher EPCs recruitment and enhanced neovascularization attained in fullthickness wounds in diabetic rats. This was due to an upregulation of VEGF and SDF-1 when compared to only ASCs and only PRP groups [70]. Interestingly, this did not seem to happen with hyaluronic acid byproducts, at least from 2 weeks after transplantation onward at which the condition only with gellan gum/hyaluronic acid showed higher vessel density than that when it was combined with ASCs [30]. This is potentially due to a residence time for the transplanted ASCs lower than 2 weeks.

Adipose tissue stromal vascular fraction (SVF) comprises, in addition to ASCs, several other cell types including fibroblasts, pre-adipocytes, ECs and hematopoietic cells [71,72]. This cell complexity present in SVF allows generating a dynamic and self-regulating angiogenic microenvironment capable of promoting the neovascularization of ischemic tissues [65]. A comparative study involving ASCs and SVF injected in full-thickness wound in diabetic mice, showed that the number of EC at the wound site was superior in the SVF group [73]. Interestingly, the angiogenic environment supported by SVF cells also permits to attain in vitro complex and interconnected capillary-like networks in the absence of extrinsic growth factors [74]. This allowed using SVF cells to generate pre-vascularized dermo-epidermal constructs that inosculated with the host tissue after implantation in a full-thickness rat model, forming abundant perfused human/rat chimeric vessels [75]. Authors underline that SVF cell composition, specially the transplanted ECs/EPCs (CD31+ cells) and the ASCs (CD90+ cells) are responsible for the generation of mature and stable capillaries [75]. Another study showed that the level of maturation of the pre-vascular network formed from SVF cells also influences the vascularization of full-thickness rat wounds $\left[76^{\circ}\right]$. Faster inosculation and superior survival of transplanted cells is linked to pericytes, whose number was significantly higher in the more mature constructs $\left[76^{\circ}\right.$ ]. A full-scale randomized clinical trial for the treatment of chronic wounds showed that pre-vascularized dermal-epidermal skin constructs using the SVF-derived endothelial cell population promote vascular network regeneration. The preliminary results published so far showed that the vascular beds of the hypodermis were denser in the pre-vascularized group, which also depicted faster wound closure [77]. These results positively support the use of SVF to enhance the vascularization of cutaneous wounds, and additional knowledge regarding 
the involved mechanisms of action will certainly allow to take a greater number of approaches in the clinical setting.

\section{Conclusion and future perspectives}

There is a pressing need to improve vascularization in skin wound healing, as it is a crucial step to promote better healing and improve neotissue quality. However, it is also clear that strategies capable of triggering controlled vascularization by vasculogenesis or angiogenesis are still a challenge. Biomaterial-based and cell-based approaches open up the possibility of engineering constructs that allow regulating angiogenesis/vasculogenesis at different levels. Biomaterial-based strategies were proven capable of regulating localized vascularization during healing through integrin-mediated responses to peptidefunctionalized biomaterials. However, its effectiveness in cutaneous wounds is still to be demonstrated and the fundamental understanding of those responses and the engagement of those peptides as angiogenic/vasculogenic initiators requires further investigation. Concomitantly, the use of angiogenic biomaterials or the release/delivery of angiogenic biomolecules or respective encoding genetic material in the wound allow to achieve a temporal effect on vascularization. While this is critical, those approaches have shown limited control due to the low dependence of the release/delivery profiles on the wound environment, and in particular on wound features such as $\mathrm{pH}$ and temperature. Therefore, the use of materials that respond to those stimuli releasing a certain biomolecule, for example, would allow responding to the specific needs of each stage of wound healing or different type of wounds. Additionally, given the temporal complexity of the wound healing process, the combination of those features with the spatial cell response mediated by integrins is likely to be relevant not only in successfully accelerating angiogenesis, critical to foster wound closure, but also to mediate the transition from the inflammatory to the proliferative phase of the healing, supporting the formation of neotissue with a quality closer to the native one. Nonetheless, new knowledge about the spatial and temporal regulation of angiogenesis during the healing of different types of wounds will also pave the way to further advance biomaterials' properties to improve effectiveness.

Pre-vascularized cellular approaches are highly effective in fostering inosculation with host tissue and consequently enhancing neovascularization. However, the availably of the ECs at clinically relevant numbers for constructs pre-vascularization has been challenging to attain. On the other side, current skin substitutes are in general effective in promoting wound healing but can be associated to delayed $t$ neo-vascularization. Knowing that the crosstalk between the epidermal cells, dermal fibroblasts and ECs, favors angiogenesis associated responses from ECs, skin substitutes might be further advanced by taking advantage of this knowledge. Advances in genetic engineering might be critical to explore of those interactions by modifying fibroblasts and/or keratinocytes to respond, as part of skin substitutes, to a specific signaling or to secrete a molecule that directly interacts with host ECs. Moreover, this vision in combination with what was discussed from the biomaterial-based perspective, can also foster the development of improved GAMs.

From the perspective of having cellular approaches with low or none cell manipulation, adipose tissue must be considered a highly relevant source of cells. It is well demonstrated that the ASCs angiogenic secretome positively impacts the vascularization of cutaneous wounds. What is still to be better understood is how this potential can be maximized in different wounds. One might speculate that strategies that enhance ASCs residence time and support this angiogenic phenotype such as for example the use of integrin-specific biomaterials could be considered. On the other hand, due to their composition, SVF has allowed the development of pre-vascularized constructs which generate a self-regulatory angiogenic environment being therefore an extremely powerful alternative for promoting vascularization in cutaneous wounds. Notwithstanding, the associated mechanisms of action should be further investigated, to unravel their true role fostering the translation of these approaches to the clinical setting.

Overall, there are a wide range of approaches that can contribute in different ways to tackle cutaneous wound healing vascularization. Yet, their clinical relevance will be determined from the generated outputs and how deeply they respond to the ultimate need that is to promote the formation of good quality neotissue. From the vascularization point of view, that goes beyond the current analysis of the number of vessel and relies on the maturity of the generated vasculature and more importantly on the extent of blood flow provided to the neotissue to guarantee long term functionality of the skin. Moreover, with the advance of the knowledge and tools that were discussed above, it is critical that strategies are thought having in consideration the type of wound and consequently its microenvironment. Taking into play what is known about the healing progression, those might also require connection not only to different wounds but also to the stage of healing.

\section{Conflict of interest statement}

Nothing declared.

\section{CRediT authorship contribution statement}

Helena R Moreira: Conceptualization, Visualization, Writing - original draft. Alexandra P Marques: Conceptualization, Supervision, Writing - review \& editing, Project administration, Funding acquisition. 


\section{Acknowledgements}

The authors thank the financial support given by the European Research Council through the consolidator grant 'ECM_INK' (ERC-2016-COG726061) and to the FSE/POCH (Fundo Social Europeu através do Programa Operacional do Capital Humano) under the scope of the PD/169/2013, NORTE-08-5369-FSE-000037. Authors would also like to acknowledge BioRender.com as a platform for image creation.

\section{References and recommended reading}

Papers of particular interest, published within the period of review, have been highlighted as:

- of special interest

• of outstanding interest

1. Chouhan D, Dey N, Bhardwaj N, Mandal BB: Emerging and

-. innovative approaches for wound healing and skin regeneration: current status and advances. Biomaterials 2019, 216:119267

This extensive review summarizes the recent advances in wound healing and skin regeneration taking into consideration several smart technologies (in situ 3D printing, portable bioprinters, electrosprayers and in situ forming hydrogels) and numerous cellular therapies (immunomodulation, stromal vascular fraction treatments, micro RNA and small interfering RNA).

2. Kiritsy CP, Lynch SE: Role of growth factors in cutaneous wound healing: a review. Crit Rev Oral Biol Med 1994, 4:729-760.

3. Diegelmann RF, Cohen IK, Kaplan AM: The role of macrophages in wound repair: a review. Plast Reconstr Surg 1981, 68:107-113.

4. Barrientos S, Stojadinovic O, Golinko MS, Brem H, TomicCanic M: Growth factors and cytokines in wound healing. Wound Repair Regen 2008, 16.

5. Rouwkema J, Rivron NC, van Blitterswijk CA: Vascularization in tissue engineering. Trends Biotechnol 2008, 26:434-441.

6. Sen CK: Human wound and its burden: updated 2020 compendium of estimates. Adv Wound Care 2021, 10:281292.

7. Tresierra-Ayala MÁ, García Rojas A: Association between peripheral arterial disease and diabetic foot ulcers in patients with diabetes mellitus type 2. Med Univ 2017, 19:123-126.

8. Armstrong DG, Boulton AJM, Bus SA: Diabetic foot ulcers and their recurrence. N Engl J Med 2017, 376:2367-2375.

9. Lee JH, Parthiban P, Jin GZ, Knowles JC, Kim HW: Materials roles for promoting angiogenesis in tissue regeneration. Prog Mater Sci 2020, 117:100732.

10. Novosel EC, Kleinhans C, Kluger PJ: Vascularization is the key challenge in tissue engineering. Adv Drug Deliv Rev 2011, 63:300-311.

11. Sharma D, Ross D, Wang G, Jia W, Kirkpatrick SJ, Zhao F: Upgrading prevascularization in tissue engineering: a review of strategies for promoting highly organized microvascular network formation. Acta Biomater 2019, 95:112-130.

12. da Silva LP, Cerqueira MT, Sousa RA, Reis RL, Correlo VM, Marques AP: Engineering cell-adhesive gellan gum spongylike hydrogels for regenerative medicine purposes. Acta Biomater 2014, 10:4787-4797.

13. Sasagawa T, Shimizu T, Yamato M, Okano T: Endothelial colonyforming cells for preparing prevascular three-dimensional cell-dense tissues using cell-sheet engineering. J Tissue Eng Regen Med 2016, 10.

14. Baltazar T, Merola J, Catarino C, Xie CB, Kirkiles-Smith NC, Lee V, Hotta S, Dai G, Xu X, Ferreira FC et al.: Three dimensional bioprinting of a vascularized and perfusable skin graft using human keratinocytes, fibroblasts, pericytes, and endothelial cells. Tissue Eng Part A 2020, 26:227-238.

15. Medina RJ, O'Neill CL, O'Doherty TM, Chambers SEJ, GuduricFuchs J, Neisen J, Waugh DJ, Simpson DA, Stitt AW: Ex vivo expansion of human outgrowth endothelial cells leads to IL-8- mediated replicative senescence and impaired vasoreparative function. Stem Cells 2013, 31:1657-1668.

16. Muñoz-Chápuli R, Quesada AR, Medina MÁ: Angiogenesis and signal transduction in endothelial cells. Cell Mol Life Sci 2004, 61:2224-2243.

17. Johnson KE, Wilgus TA: Vascular endothelial growth factor and angiogenesis in the regulation of cutaneous wound repair. Adv Wound Care 2014, 3:647-661.

18. Kolte D, McClung JA, Aronow WS: Vasculogenesis and angiogenesis. Translational Research in Coronary Artery Disease. 2016 http://dx.doi.org/10.1016/B978-0-12-802385-3.00006-1.

19. Demidova-Rice TN, Durham JT, Herman IM: Wound healing angiogenesis: innovations and challenges in acute and chronic wound healing. Adv Wound Care 2012, 1:17.

20. Zeng Y, Zhu L, Han Q, Liu W, Mao X, Li Y, Yu N, Feng S, Fu Q, Wang $X$ et al.: Preformed gelatin microcryogels as injectable cell carriers for enhanced skin wound healing. Acta Biomater 2015, 25.

21. Malinda KM, Wysocki AB, Koblinski JE, Kleinman HK, Ponce ML: Angiogenic laminin-derived peptides stimulate wound healing. Int J Biochem Cell Biol 2008, 40:2771.

22. Martino MM, Briquez PS, Ranga A, Lutolf MP, Hubbell JA: Heparin-binding domain of fibrin(ogen) binds growth factors and promotes tissue repair when incorporated within a synthetic matrix. Proc Natl Acad Sci U S A 2013, 110:4563-4568.

23. Wang W, Guo L, Yu Y, Chen Z, Zhou R, Yuan Z: Peptide REDVmodified polysaccharide hydrogel with endothelial cell selectivity for the promotion of angiogenesis. J Biomed Mater Res Part A 2015, 103:1703-1712.

24. Li S, Nih LR, Bachman H, Fei P, Li Y, Nam E, Dimatteo R,

- Carmichael ST, Barker TH, Segura T: Hydrogels with precisely controlled integrin activation dictate vascular patterning and permeability. Nat Mater 2017, 16:953-961

This study showed the importance of integrin engagement of angiogenic cells to biomaterials and the resultant formation of non-leaky/leaky vesse formation attributed to the expression of VE-cadherin at cell-cell junctions.

25. Rowland FN, Donovan MJ, Picciano PT, Wilner GD, Kreutzer DL: Fibrin-mediated vascular injury: identification of fibrin peptides that mediate endothelial cell retraction. Am J Pathol 1984, 117:418-428.

26. Ge M, Ryan TJ, Lum H, Malik AB: Fibrinogen degradation product fragment $D$ increases endothelial monolayer permeability. Am J Physiol 1991, 261:L283-9.

27. da Silva LP, Pirraco RP, Santos TC, Novoa-Carballal R,

- Cerqueira MT, Reis RL, Correlo VM, Marques AP. Neovascularization induced by the hyaluronic acid-based spongy-like hydrogels degradation products. ACS Appl Mater Interfaces 2016, 8:33464-33474

This study showed the importance of the byproducts of degradation of the hyaluronic acid after implantation in an ischemic hind limb. The materials promoted neovascularization in a material-dependent manner and sustained release, consistent with the in vitro degradation profile.

28. Jarrell DK, Vanderslice EJ, Lennon ML, Lyons AC, VeDepo MC, Jacot JG: Increasing salinity of fibrinogen solvent generates stable fibrin hydrogels for cell delivery or tissue engineering PLoS One 2021, 16

29. Segura $T$, Anderson BC, Chung PH, Webber RE, Shull KR, Shea LD: Crosslinked hyaluronic acid hydrogels: a strategy to functionalize and pattern. Biomaterials 2005, 26:359-371.

30. da Silva LP, Santos TC, Rodrigues DB, Pirraco RP, Cerqueira MT, Reis RL, Correlo VM, Marques AP: Stem cell-containing hyaluronic acid-based spongy hydrogels for integrated diabetic wound healing. J Invest Dermatol 2017, 137:1541-1551.

31. Traub S, Morgner J, Martino M, Höning S, Swartz M, Wickström S, Hubbell J, Eming S: The promotion of endothelial cell attachment and spreading using FNIII10 fused to VEGF-A165. Biomaterials 2013, 34:5958-5968. 
32. Matsumoto S, Tanaka R, Okada K, Arita K, Hyakusoku H, Miyamoto M, Tabata Y, Mizuno H: The effect of control-released basic fibroblast growth factor in wound healing: Histological analyses and clinical application. Plast Reconstr Surg 2013, 1 e44.

33. Olekson MAP, Faulknor R, Bandekar A, Sempkowski M, Hsia HC Berthiaume F: SDF-1 liposomes promote sustained cell proliferation in mouse diabetic wounds. Wound Repair Regen 2015, 23:711-723.

34. Park TY, Maeng SW, Jeon EY, Joo KII, Cha HJ: Adhesive proteinbased angiogenesis-mimicking spatiotemporal sequential release of angiogenic factors for functional regenerative medicine. Biomaterials 2021, 272:120774.

35. Vijayan A, S A, Kumar GSV: PEG grafted chitosan scaffold for dual growth factor delivery for enhanced wound healing. Sci Rep 2019, 9:1-12.

36. Qu Y, Cao C, Wu Q, Huang A, Song Y, Li H, Zuo Y, Chu C, Li J, Man $Y$ : The dual delivery of KGF and bFGF by collagen membrane to promote skin wound healing. J Tissue Eng Regen Med 2018, 12:1508-1518.

37. Long G, Liu D, He X, Shen Y, Zhao Y, Hou X, Chen B, OuYang W Dai J, Li X: A dual functional collagen scaffold coordinates angiogenesis and inflammation for diabetic wound healing. Biomater Sci 2020, 8:6337-6349.

38. Ishihara J, Ishihara A, Fukunaga K, Sasaki K, White MJV,

- Briquez PS, Hubbell JA: Laminin heparin-binding peptides bind to several growth factors and enhance diabetic wound healing. Nat Commun 2018, 9:1-14

This work showed the multifunctional laminin heparin-binding domains to as a growth factor retention strategy that enhances the efficacy of vascular endothelial cell growth factor (VEGF-A165) and platelet-derived growth factor (PDGF-BB) in promoting wound healing in vivo.

39. Raftery RM, Tierney EG, Curtin CM, Cryan S-AA, O’Brien FJ: Development of a gene-activated scaffold platform for tissue engineering applications using chitosan-pDNA nanoparticles on collagen-based scaffolds. J Control Release 2015, 210:8494.

40. Lugano R, Ramachandran M, Dimberg A: Tumor angiogenesis: causes, consequences, challenges and opportunities. Cell Mol Life Sci 2019, 77:1745-1770.

41. Curtin CM, Tierney EG, Mcsorley K, Cryan SA, Duffy GP, O'Brien FJ: Combinatorial gene therapy accelerates bone regeneration: non-viral dual delivery of VEGF and BMP2 in a collagen-nanohydroxyapatite scaffold. Adv Healthc Mater 2015, 4:223-227.

42. Lou D, Luo Y, Pang Q, Tan WQ, Ma L: Gene-activated dermal equivalents to accelerate healing of diabetic chronic wounds by regulating inflammation and promoting angiogenesis. Bioact Mater 2020, 5:667-679.

43. Wang P, Huang S, Hu Z, Yang W, Lan Y, Zhu J, Hancharou A Guo R, Tang B: In situ formed anti-inflammatory hydrogel loading plasmid DNA encoding VEGF for burn wound healing. Acta Biomater 2019, 100:191-201.

44. Hanjaya-Putra D, Bose V, Shen YI, Yee J, Khetan S, Fox-Talbot K, Steenbergen C, Burdick JA, Gerecht S: Controlled activation of morphogenesis to generate a functional human microvasculature in a synthetic matrix. Blood 2011, 118:804815.

45. da Silva LP, Jha AK, Correlo VM, Marques AP, Reis RL, Healy KE:

- Gellan gum hydrogels with enzyme-sensitive biodegradation and endothelial cell biorecognition sites. Adv Healthc Mater 2018, 7:1700686

This manuscript describes the use of chemically functionalized materials with different angiogenic peptides and a peptide crosslinker sensitive to MMP-1 for MMP-1-driven degradation for endothelial cell biorecognition cues. This is a promising endothelial cell responsive materials strategy that can be used for vascularization strategies.

46. Smola H, Thiekotter G, Fusenig NE: Mutual induction of growth factor gene expression by epidermal-dermal cell interaction. Cell Biol 1993, 122:417-429.
47. Xia YP, Zhao Y, Marcus J, Jimenez PA, Ruben SM, Moore PA, Khan F, Mustoe TA: Effects of keratinocyte growth factor-2 (KGF-2) on wound healing in an ischaemia-impaired rabbit ear model and on scar formation. J Pathol 1999, 188:431-438.

48. Ziche M, Morbidelli L, Choudhuri R, Zhang HT, Donnini S, Granger HJ, Bicknell R: Nitric oxide synthase lies downstream from vascular endothelial growth factor-induced but not basic fibroblast growth factor-induced angiogenesis. J Clin Invest 1997, 99:2625.

49. Supp DM, Supp AP, Bell SM, Boyce ST: Enhanced vascularization of cultured skin substitutes genetically modified to overexpress vascular endothelial growth factor. $J$ Invest Dermatol 2000, 114:5-13.

50. Supp DM, Boyce ST: Overexpression of vascular endothelial growth factor accelerates early vascularization and improves healing of genetically modified cultured skin substitutes. Burn Care Rehabil 2002, 23:10-20.

51. Mitchell K, Szekeres C, Milano V, Svenson KB, Nilsen-Hamilton M, Kreidberg JA, DiPersio CM: Alpha3beta1 integrin in epidermis promotes wound angiogenesis and keratinocyte-toendothelial-cell crosstalk through the induction of MRP3. J Cell Sci 2009, 122:1778-1787.

52. Detmar M: The role of VEGF and thrombospondins in skin angiogenesis. J Dermatol Sci 2000, 24(Suppl. 1):S78-84.

53. Varkey M, Ding J, Tredget E: Fibrotic remodeling of tissueengineered skin with deep dermal fibroblasts is reduced by keratinocytes. Tissue Eng Part A 2014, 20:716-727.

54. Grøn B, Stoltze K, Andersson A, Dabelsteen E: Oral fibroblasts produce more HGF and KGF than skin fibroblasts in response to co-culture with keratinocytes. APMIS 2002, 110:892-898.

55. Ayata RE, Chabaud S, Auger M, Pouliot R: Behaviour of endothelial cells in a tridimensional in vitro environment. Biomed Res Int 2015, 2015.

56. Caneparo C, Baratange C, Chabaud S, Bolduc S: Conditioned medium produced by fibroblasts cultured in low oxygen pressure allows the formation of highly structured capillarylike networks in fibrin gels. Sci Rep 2020, 10:1-11.

57. Stunova A, Vistejnova L: Dermal fibroblasts - a heterogeneous

-. population with regulatory function in wound healing. Cytokine Growth Factor Rev 2018, 39:137-150

This review describes the capacity of dermal fibroblasts to sense and respond to signals from the microenvironment and to communicate with surrounding cells during cutaneous wound healing. The review further emphasizes the, to date, poorly understood roles of heterogeneous dermal fibroblast populations in the wound healing process.

58. Newman AC, Nakatsu MN, Chou W, Gershon PD, Hughes CCWW: The requirement for fibroblasts in angiogenesis: fibroblastderived matrix proteins are essential for endothelial cell lumen formation. Mol Biol Cell 2011, 22:3791-3800.

59. Berthod F, Germain L, Tremblay N, Auger FA: Extracellular matrix deposition by fibroblasts is necessary to promote capillarylike tube formation in vitro. J Cell Physiol 2006, 207:491-498.

60. Sorrell JM, Baber MA, Caplan Al: Human dermal fibroblast subpopulations; differential interactions with vascular endothelial cells in coculture: nonsoluble factors in the extracellular matrix influence interactions. Wound Repair Regen 2008, 16:300-309

61. Philippeos C, Telerman SB, Oulès B, Pisco AO, Shaw TJ, Elgueta R, Lombardi G, Driskell RR, Soldin M, Lynch MD et al.: Spatial and single-cell transcriptional profiling identifies functionally distinct human dermal fibroblast subpopulations. J Invest Dermatol 2018, 138:811-825.

62. Ong WK, Chakraborty S, Sugii S: Adipose tissue: understanding the heterogeneity of stem cells for regenerative medicine. Biomolecules 2021, 11:918.

63. Melchiorri AJ, Nguyen BNB, Fisher JP: Mesenchymal stem cells: roles and relationships in vascularization. Tissue Eng Part $B$ Rev 2014, 20:218-228. 
64. Laschke MW, Kleer S, Scheuer C, Schuler S, Garcia P, Eglin D, Alini M, Menger MD: Vascularisation of porous scaffolds is improved by incorporation of adipose tissue-derived microvascular fragments. Eur Cells Mater 2012, 24:266-277.

65. Rehman J, Traktuev D, Li J, Merfeld-Clauss S, Temm-Grove CJ, Bovenkerk JE, Pell CL, Johnstone BH, Considine RV, March KL: Secretion of angiogenic and antiapoptotic factors by human adipose stromal cells. Circulation 2004, 109:1292-1298.

66. Cerqueira MT, Pirraco RP, Santos TC, Rodrigues DB, Frias AM, Martins AR, Reis RL, Marques AP: Human adipose stem cells cell sheet constructs impact epidermal morphogenesis in fullthickness excisional wounds. Biomacromolecules 2013, 14:3997-4008.

67. Fujiwara O, Prasai A, Perez-Bello D, El Ayadi A, Petrov I, Esenaliev R, Petrov Y, Herndon D, Finnerty C, Prough D et al. Adipose-derived stem cells improve grafted burn wound healing by promoting wound bed blood flow. Burn trauma 2020 8:tkaa009.

68. Nagano H, Suematsu Y, Takuma M, Aoki S, Satoh A, Takayama E, Kinoshita M, Morimoto Y, Takeoka S, Fujie T et al.: Enhanced cellular engraftment of adipose-derived mesenchymal stem cell spheroids by using nanosheets as scaffolds. Sci Rep 2021 , 11:1-12.

69. Burmeister DM, Stone R II, Wrice N, Laborde A, Becerra SC, Natesan S, Christy RJ: Delivery of allogeneic adipose stem cells in polyethylene glycol-fibrin hydrogels as an adjunct to meshed autografts after sharp debridement of deep partial thickness burns. Stem Cells Transl Med 2018, 7:360.

70. Ebrahim N, Dessouky AA, Mostafa O, Hassouna A, Yousef MM, Seleem Y, El Gebaly EAEAM, Allam MM, Farid AS, Saffaf BA et al.: Adipose mesenchymal stem cells combined with platelet-rich plasma accelerate diabetic wound healing by modulating the Notch pathway. Stem Cell Res Ther 2021, 12:1-24.

71. Planat-Benard V, Silvestre J-S, Cousin B, Andre' M, Nibbelink M, Tamarat R, Clergue M, Manneville C, Saillan-Barreau C, Duriez M et al.: Plasticity of human adipose lineage cells toward endothelial cells. Circulation 2004, 109:656-663.
72. Yoshimura K, Shigeura T, Matsumoto D, Sato T, Takaki Y, AibaKojima E, Sato K, Inoue K, Nagase T, Koshima I et al.: Characterization of freshly isolated and cultured cells derived from the fatty and fluid portions of liposuction aspirates. J Cell Physiol 2006, 208:64-76.

73. Bi H, Li H, Zhang C, Mao Y, Nie F, Xing Y, Sha W, Wang X, Irwin DM, Tan $\mathrm{H}$ : Stromal vascular fraction promotes migration of fibroblasts and angiogenesis through regulation of extracellular matrix in the skin wound healing process. Stem Cell Res Ther 2019, 10:302.

74. Costa M, Cerqueira MT, Santos TC, Sampaio-Marques B, Ludovico P, Marques AP, Pirraco RP, Reis RL: Cell sheet engineering using the stromal vascular fraction of adipose tissue as a vascularization strategy. Acta Biomater 2017, 55.

75. Klar AS, Güven S, Zimoch J, Zapiórkowska NA, Biedermann T, Böttcher-Haberzeth S, Meuli-Simmen C, Martin I, Scherberich A Reichmann E et al.: Characterization of vasculogenic potential of human adipose-derived endothelial cells in a threedimensional vascularized skin substitute. Pediatr Surg Int 2016, 32:17-27.

76. Cerino G, Gaudiello E, Muraro MG, Eckstein F, Martin I,

- Scherberich A, Marsano A: Engineering of an angiogenic niche by perfusion culture of adipose-derived stromal vascular fraction cells. Sci Rep 2017, 7:14252

This manuscript described the use of stromal vascular fraction cultured under flow and static conditions. Only perfusion culture promoted in vitro the early formation of a capillary-like network, embedded within an ECM backbone, and the release of numerous pro-angiogenic factors, due to the number of pericytes that led to increased maturation of the capillarylike structures formed in vitro.

77. Nilforoushzadeh MA, Sisakht MM, Amirkhani MA, Seifalian AM, Banafshe HR, Verdi J, Nouradini M: Engineered skin graft with stromal vascular fraction cells encapsulated in fibrin-collagen hydrogel: a clinical study for diabetic wound healing. $J$ Tissue Eng Regen Med 2020, 14:424-440. 